\title{
Incoordination, Paralysis and Recovery after Pyrethroid Treatment on Nymphs III of Triatoma infestans (Hemiptera: Reduviidae)
}

\author{
Raúl A Alzogaray, Eduardo N Zerba ${ }^{+}$ \\ Centro de Investigaciones de Plagas e Insecticidas, (CIPEIN-CITEFA/ CONICET), Zufriategui 4380, \\ 1603 Villa Martelli, Buenos Aires, Argentina
}

\begin{abstract}
Symptoms of poisoning for deltamethrin and cis-permethrin on nymphs III of Triatoma infestans were described. The time required for incoordination and paralysis were determined. Deltamethrin was equal or more rapid in the onset of the first effect (accordingly to dose), and cis-permethrin in the onset of the second one. There were no significant differences between incoordination doses $50 \%\left(\right.$ IncD $\left._{50} s\right)$ at different times for the two pyrethroids. They showed equivalent incoordination power, but the nymphs treated with deltamethrin recovered slightly more rapid and in greater amount than the nymphs treated with cis-permethrin. The recovery was inhibited by the simultaneus application of piperonyl butoxide. This result suggests that biotransformation by mixed-function microsomal oxidases are involved in the process of recovery.
\end{abstract}

Key words: Triatoma infestans - deltamethrin - cis-permethrin - pyrethroids - poisoning symptoms

The bloodsucking bug Triatoma infestans is one of the more important vectors of Chagas' disease in South America (WHO 1991), and the pyrethroids are the more effective triatomicides (Casabé et al. 1988). These neurotoxic insecticides are an effective tool in national control programs in Argentina (Zerba 1988). Particularly, deltamethrin is a cyanopyrethroid broadly used in control programs (Zerba 1989). Permethrin is an isomers mixture well established against public health pests because of its high insecticidal power and low mammalian toxicity (WHO 1990), but it is not usually used in triatomine control because of its unfavourable cost-benefit relationship. Previous works by our laboratory showed that the $c i s$ isomer of permethrin have an important insecticidal activity on nymphs I of $T$. infestans (Alzogaray \& Zerba 1996). On the other hand, it has been demonstrated that the less active trans isomer produces an antagonistic effect which masks the high toxicity of the cis isomer when they are simultaneously applied on both nymphs

\footnotetext{
This investigation received financial support from the UNDP/World Mundial Bank/WHO Special Programme for Research and Trainning in Tropical Diseases, from the Consejo Nacional de Investigaciones Científicas y Técnicas of Argentina (CONICET), and from Chemotecnica Sintyal (Argentina).

+Corresponding author. Fax: +54-1-709.3210

Received 27 May 1996

Accepted 19 February 1997
}

III and V of T. infestans (Alzogaray et al. unpublished results). These results suggest that cispermethrin has potential use in the control of Chagas' disease vectors. The specific aim of the present work was to compare the onset of incoordination, paralysis and recovery by deltamethrin and cis-permethrin on nymphs III of $T$. infestans. This knowledge could be relevant to analyze a possible use of cis-permethrin in future measures for Chagas' disease vectors control.

\section{MATERIALS AND METHODS}

Biological material - Nymphs III of T. infestans were obtained from a colony mantained in our laboratory at $28^{\circ} \mathrm{C}$ and a photoperiod of $12: 12$ (L: D) hr. The experiments were done on 7-11 days old nymphs starved from the last moult.

Chemicals - Deltamethrin [(S)- $\alpha$-cyano-3phenoxybenzyl (1R)-cis-3-(2,2-dibromovynil)2,2-dimethylcyclopropanecarboxylate] (97\%) was provided by courtesy of Roussel-Uclaf (France). Cis-permethrin [3-phenoxybenzyl (1RS)-cis-3-(2, 2-dichlorovynil)-2,2-dimethylcyclopropanecarboxylate] (99\%) was a gift from Chemotecnica Sintyal (Argentina).

Symptomatology - Pyrethroids were topically applied on the ventral side of the abdominal surface in $0.2 \mu \mathrm{l}$ of an acetonic solution, each insect receiving $0.02 \mu \mathrm{g}$ or $2 \mu \mathrm{g}$ of insecticide. The nature and order of appearance of the symptoms of poisoning were recorded by individual observation of at least 10 nymphs per dose. The following sequence of symptoms was observed: (1) sligth 
tremors; (2) backward or side displacement; (3) contraction of the three pairs of legs beneath the body; (4) elevation above the body plane; (5) paralysis of the third pair of legs; (6) violent convulsive movements; (7) paralysis of the three pairs of legs; (8) slow or occasional movements; (9) lack of movements.

Bioassays - Pyrethroids were topically applied on the ventral side of the abdominal surface in $0.2 \mu \mathrm{l}$ of an acetonic solution. Control groups were topically treated with acetone alone. Immediately after topical application the nymphs were placed in a constant temperature chamber at $26^{\circ} \mathrm{C}(60-90 \%$ $\mathrm{RH})$.

To calculate the time required for $50 \%$ of the experimental population to show incoordination or paralysis ( $\mathrm{IT}_{50}$ or $\mathrm{PT}_{50}$, respectively), groups of 5 nymphs were treated topically with a single dose of pyrethroid in acetone. The experiments were performed with either 0.02 or $2 \mu \mathrm{g} / \mathrm{insect}$. The first of these doses is approximately the incoordination dose $99 \%$ (IncD 99) for both the pyrethroids assayed. The evaluated effects were (1) incoordination, defined as the incapacity to leave a filter paper disc of $15 \mathrm{~cm}$ diameter in tenfold time required by controls (which is $<5 \mathrm{sec}$ ); and (2) paralysis, defined as lack of movements in legs, antennae and proboscis. The appearance of these effects was followed as a function of the time after the treatment. Each experiment was replicated four times.

Doses between 2 and $128 \mathrm{ng} /$ insect were applied to groups of five nymphs to calculate the $\mathrm{IncD}_{50}$. Nymphs were examined for incoordination at different times after insecticide application in order to establish the dose-response relationship. In this way, an $\mathrm{IncD}_{50}$ value for each time was obtained. The bioassays were interrupted at $21 \mathrm{st}$ day, when the control mortality was greater than $10 \%$. Each experiment was replicated twice $(\mathrm{N}=$ 40-60).

The values of $\mathrm{IT}_{50}, \mathrm{PT}_{50}$ or $\mathrm{IncD}_{50}$ were calculated using the probit method (Litchfield \& Wilcoxon 1949). Differences between values were considered significant $(P<0.05)$ if the respective 95\% CL did not overlap (Toth \& Sparks 1988).

Filter papers Whatman \# 1 were impregnated with a solution of piperonyl butoxide in acetone $\left(0.07 \mathrm{mg} / \mathrm{cm}^{2}\right)$. The nymphs were permanently exposed to the treated papers during each experiment. Control groups were exposed to filter papers treated with acetone alone.

Incoordination and recovery analysis - $\mathrm{IncD}_{50}$ versus time curves were made to show the distinct phases described by Sawicki (1962) on the housefly Musca domestica: a falling line (phase 1), a ris- ing line (phase 2), and a plateau (phase 3). This way to present the results allow to see how the proportion of affected insects at each dose increases as a function of the time after the insecticide application, and it reaches a minimum value (the Incoordination Endpoint - IE). After IE is reached, the curve increases while the insects recovery from the temporary incoordination. Finally, the recovery stops and a plateau is observed. The Effectivity: Incoordination Ratio and the Incoordination Recovery Rate (IRR) were calculated based in Sawicki (1962). Effectivity: Incoordination Ratio $=\mathrm{IncD}_{50 \mathrm{p} 3} / \mathrm{IncD}_{50 \mathrm{ep}} ;$ where $\mathrm{IncD}_{50 \mathrm{p} 3}$ is the $\operatorname{IncD}_{50}$ in the phase 3 of the curve, and IncD ep is the minimum $\mathrm{IncD}_{50}$ observed before recovery occurs (IE). IRR is the slope of the phase 2 of the curve and it is expressed as ng/nymph/day. The Effectivity: Inccordination Ratio is a measure of how much recovery occurs. The IRR is a measure of how rapidly the recovery occurs.

\section{RESULTS AND DISCUSSION}

The symptoms of intoxication by pyrethroids observed in T. infestans (see Materials and Methods) have been reported by other authors in the cockroach Periplaneta americana (Gammon et al. 1981, Benoit et al. 1985), and Musca domestica (Adams \& Miller 1980). A correlation between visible and neurophysiological events of poisoning by allethrin have been established in $P$. americana (Gammon 1978).

Results in Table I show that the values of $\mathrm{IT}_{50}$ were inversely proportional to the dose for the two pyrethroids assayed. When a dose of $0.02 \mu \mathrm{g} / \mathrm{in}$ sect was applied, the $\mathrm{IT}_{50}$ for both pyrethroids were not significantly different (based on overlap of $95 \%$ $\mathrm{CL}, P>0.05)$. In change, when the dose was $2 \mu \mathrm{g} /$ insect, the $\mathrm{IT}_{50}$ for deltamethrin was significantly smaller than the $\mathrm{IT}_{50}$ for cis-permethrin (based on nonoverlap of $95 \% \mathrm{CL}, P<0.05$ ).

TABLE I

$\mathrm{IT}_{50}{ }^{a}$ of deltamethrin and cis-permethrin on nymphs of Triatoma infestans

\begin{tabular}{ccc}
\hline \multirow{2}{*}{$\begin{array}{c}\text { Dose } \\
(\mu \mathrm{g} / \mathrm{insect})\end{array}$} & \multicolumn{2}{c}{$\mathrm{IT}_{50}{ }^{a}(95 \% \mathrm{CL})^{b}(\mathrm{~min})$} \\
\cline { 2 - 3 } & deltamethrin & cis-permethrin \\
\hline 0.02 & 454.8 & 494.5 \\
& $(409.0-525.0)$ & $(474.6-520.9)$ \\
2 & 77.8 & 138.7 \\
& $(65.1-89.0)$ & $(124.6-150.1)$ \\
\hline
\end{tabular}

Each value was calculated from the data obtained in four independent replicates.

$a$ : Incoordination Time 50\%; $b$ : 95\% Confidence Limits. 
Results in Table II show the values of $\mathrm{PT}_{50}$ for the two insecticides. For the same dose, the nymphs treated with cis-permethrin showed lack of movements in a time significantly smaller than the nymphs treated with deltamethrin (based on nonoverlap 95\% CL, $P<0.05$ ). Not significant differences were observed for $\mathrm{PT}_{50}$ produced by different doses of a same insecticide (based on overlap of $95 \% \mathrm{CL}, P>0.05)$.

\section{TABLE II}

$\mathrm{PT}_{50}{ }^{a}$ of deltamethrin and cis-permethrin on nymphs III of Triatoma infestans

\begin{tabular}{ccc}
\hline & \multicolumn{2}{c}{$\mathrm{PT}_{50}{ }^{a}(95 \% \mathrm{CL})^{b}$ (days) } \\
\cline { 2 - 3 } $\begin{array}{c}\text { Dose } \\
\mu \mathrm{g} / \text { insect })\end{array}$ & deltamethrin & cis-permethrin \\
\hline 0.02 & 22.7 & 7.8 \\
& $(21.0-23.8)$ & $(7.8-8.7)$ \\
2 & 21.3 & 7.8 \\
& $(20.3-22.5)$ & $(6.6-8.7)$ \\
\hline
\end{tabular}

Each value was calculated from the data obtained in four independent replicates.

$a$ : Paralysis Time 50\%; $b$ : 50\% Confidence Limits.

Following the suggestion of Burt and Goodchild (1974) that the central nervous system is the site where the pyrethroids act to induce incoordination in insects, Briggs et al. (1976) concluded that rapid incoordination must involve fast and efficient penetration to the central nervous system. This was contrasted with kill, which can occur two days or more after application. These authors suggested that an important property determining whether a particular pyrethroid will be effective for incoordination or kill will be the overall polarity of the molecule, which they considered to have a marked influence on the rate at which the pyrethroid would penetrate into the site of action. Briggs and coworkers observed that among pyrethroids the optimum polarity for incoordination is slightly on the lipophobic side of that for kill, and suggested that rapid penetration, which they assumed the more polar pyrethroids can achieve, is required for incoordination.

Clements and May (1977) suggested that knockdown by pyrethroids results from a particular type of physiological action, and that the ability to induce that action is dependent upon molecular structure. These authors demonstrated that the $\alpha$-cyano moiety enhances knockdown activity of the pyrethroids.

The two hypothesis above mentionated are not mutually exclusive. In fact, our results are according to both of them.
Figure shows the variation of $\mathrm{IncD}_{50}$ for deltamethrin and cis-permethrin as a function of the time after the insecticide application, with and without piperonyl butoxide. In the curve for $\mathrm{cis}$ permethrin (Fig. b) the three phases described for pyrethrum extract in M. domestica (Sawicki 1962) were observed: rapidly falling line (phase 1), gradually rising line (phase 2), and attaining a plateau (phase 3). In the curve for deltamethrin (Fig. a), only the two first phases were observed. Before the phase 3 (a plateau) was reached, the bioassays were interrupted because of the mortality of controls was greater than $10 \%$ (at the 21 st day).
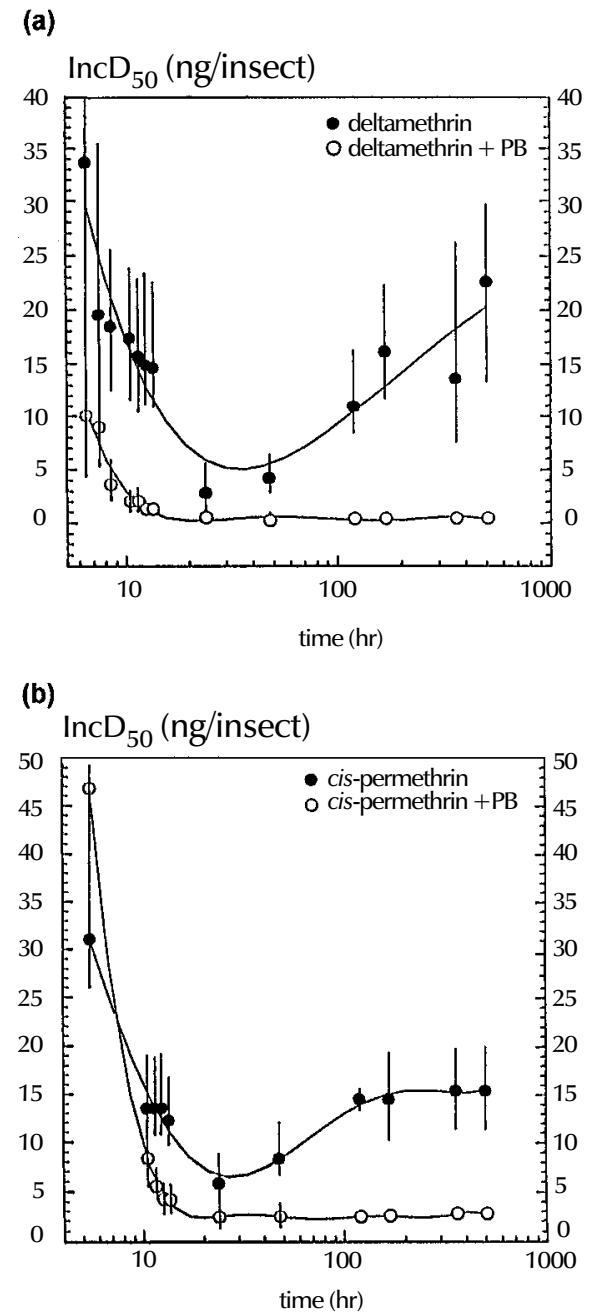

Incoordination Doses $50 \%\left(\mathrm{IncD}_{50}\right)$ versus time curves for topically applied (a) deltamethrin and (b) cis-permethrin, with or without piperonyl butoxide, on nymphs III of Triatoma infestans. The nymphs were permanently exposed to filter paper treated with a solution of piperonyl butoxide in acetone $\left(0.07 \mathrm{mg} / \mathrm{cm}^{2}\right)$ or acetone only (controls). Each value was calculated from the data obtained in two independent replicates $(\mathrm{N}=40-60)$. Bars represent the $95 \%$ confidence limit. 
The comparison of $\mathrm{IncD}_{50}$ for the two insecticides in each time indicates that there were no significant differences between the effects of deltamethrin and cis-permethrin in any time (based on overlap 95\% CL, $P>0.05$ ).

Table III shows the toxicological parameters calculated from the curves in Fig. For the two insecticides assayed, the $\operatorname{IncD}_{50}(24 \mathrm{hr})$ coincided with the IE. The IRR for deltamethrin was greater than for cis-permethrin ( 0.67 and $0.55 \mathrm{ng} / \mathrm{nymph} /$ day, respectively). The Effectivity: Incoordination Ratio was at least threefold for deltamethrin than for cis-permethrin ( $\geq 8.1$ and 2.7, respectively). These results mean that the two pyrethroids showed similar inccordination power, but the nymphs treated with deltamethrin recovery slightly more rapid and in greater amount than those treated with cis-permethrin.

\section{TABLE III}

Toxicity parameters of deltamethrin and cispermethrin on nymphs III of Triatoma infestans

\begin{tabular}{lcc}
\hline & deltamethrin & cis-permethrin \\
\cline { 2 - 3 } $\begin{array}{l}\text { Incoordination } \\
\text { Endpoint (hr) }\end{array}$ & 24 & 24 \\
$\begin{array}{l}\text { Incoordination Recovery } \\
\text { (ng/nymph/day) }\end{array}$ & 0.67 & 0.55 \\
$\begin{array}{l}\text { Effectivity: } \\
\text { Incoordination Ratio }\end{array}$ & $\geq 8.1$ & \\
\hline
\end{tabular}

When piperonyl butoxide was applied, recovery was not observed in nymphs treated with either deltamethrin or cis-permethrin (Fig.). These results indicate that the activity of mixed-function microsomal oxidases are involved in the process of recovery.

The knockdown effect is an important part of the pyrethroid action. It is rapid in onset, sometimes very long lasting, and not necessarily associated to lethal action (Naumann 1990). The three phases in the curve $\mathrm{KD}_{50}$ vs log time were described for pyrethrum and a number of pyrethroids in M. domestica (Sawicki 1962, Scott \& Georghiu 1984). In these cases, the knockdown ocurred few minutes after the application of the insecticides, and the $\mathrm{KD}_{50}$ reached a plateau near the $24 \mathrm{th} \mathrm{hr}$. Our results indicate that the events of incoordination and recovery present a very different kinetic in T. infestans. Penetration, metabolic degradation, and interaction of the insecticides with the site of action are factors known to influence the knockdown (Ruigt 1985). However, it is not clear if knockdown and kill are or not different manifes- tations of the same underlying mechanism.

The degradation at the site of action was proposed to explain the rapid recovery in $M$. domestica (Sawicki 1962). This hypothesis was supported by the lack of housefly recovery in the presence of piperonyl butoxide, an inhibitor of the activity of mixed-function microsomal oxidases. Similar results were reported in adults of $T$. infestans treated with deltamethrin and piperonyl butoxide (Casabé et al. 1988).

Previous result by our laboratory (Alzogaray \& Zerba 1996, Alzogaray et al., unpublished results) and the evidence presented in this paper indicate that $c i s$-permethrin can be considered a potential tool for the Chagas' disease vector control, and more investigations in relation with its properties should be done.

\section{REFERENCES}

Adams ME, Miller TA 1980. Neural and behavioral correlates of pyrethroid and DDT-type poisoning in the house fly, Musca domestica L. Pestic Biochem Physiol 13: 137-147.

Alzogaray RA, Zerba EN 1996. Comparative toxicity of deltamethrin and cis-permethrin on first instars of Triatoma infestans (Hemiptera: Reduviidae). J Med Entomol 33: 58-62.

Benoit M, Bruneau P, Meinard C 1985. Comparative activity of tralomethrin and deltamethrin on Periplaneta americana. Pestic Biochem Physiol 26: 284-291.

Briggs GG, Elliot M, Farnahm AW, Janes NF, Needham PH, Pulman DA, Young SR 1976. Insecticidal activity of the pyrethrins and related compounds. VIII. Relation of polarity with activity in pyrethroids. Pestic Sci 7: 236-240.

Burt PE, Goodchild RE 1974. Knockdown by pyrethroids: its role in the intoxication process. Pestic Sci 5: 625-633.

Casabé N, Melgar F, Wood EJ, Zerba EN 1988. Insecticidal activity of pyrethroids against Triatoma infestans. Insect Sci Applic 9: 233-236.

Clements AN, May TE 1977. The actions of pyrethroids upon the peripheral nervous system and associated organs in the locust. Pestic Sci 8: 661-680.

Gammon DW 1978. Neural effects of allethrin on the free walking cockroach Periplaneta americana: an investigation using defined doses at 15 and $32^{\circ} \mathrm{C}$. Pestic Sci 9: 79-81.

Gammon DW, Brown MA, Casida JE 1981. Two classes of pyrethroid action in the cockroach. Pestic Biochem Physiol 15: 181-191.

Litchfield JT, Wilcoxon FJ 1949. A simplified method of evaluating dose-effect experiments. J Exper Ther 96: 99-133.

Naumann K 1990. Synthetic pyrethroid insecticide. Springer, Berlin, viii +241 pp.

Ruigt GSF 1985. Pyrethroids, p. 183-262. In GA Kerkut, LI Gilbert (eds), Comprehensive Insect Physiology, Biochemistry and Pharmacology, vol. 12. Pergamon, Oxford. 
Sawicki RM 1962. Insecticidal activity of pyrethrum extract and its four insecticidal constituents against house fly. III. Knock-down and recovery of flies treated with pyrethrum extract with and without piperonyl butoxide. J Sci Food Agric 13: 283-292.

Scott JG, Georghiou GP 1984. Influence of temperature on knockdown, toxicity, and resistance to pyrethroids in the house fly, Musca domestica. Pestic Biochem Physiol 21: 53-62.

Toth SJ, Sparks TC 1988. Influence of treatment technique on the temperature-toxicity relationships of $\mathrm{cis}$ - and trans-permethrin in the cabbage looper (Lepidoptera: Noctuidae). J Econ Entomol 81: 115-118.

WHO - World Health Organization 1990. Permethrin. WHO, Geneva, vi + 95 pp

WHO - World Health Organization 1991. Control of Chagas disease. WHO, Geneva, vi + 93 pp.

Zerba EN 1988. Insecticidal activity of pyrethroids on insects of medical importance. Parasitol Today 4: 53-57.

Zerba EN 1989. Chemical Control of Chagas Disease Vectors. Biomed Environ Sci 2: 24-29. 
